\title{
Yod
}

Revue des études hébraïques et juives

17 | 2012

La presse écrite au Moyen-Orient

\section{La presse hébraïque, un vecteur de l'entrée des Juifs dans la modernité}

The Press as a Vehicle in the Modernisation of the Jews

$$
\text { העיתונות כגורם מוביל בתהליך המודרניזציה של היהודים }
$$

\section{Rina Cohen Muller}

\section{(2) OpenEdition}

Journals

Édition électronique

URL : https://journals.openedition.org/yod/1575

DOI : 10.4000/yod. 1575

ISSN : 2261-0200

\section{Éditeur}

INALCO

Édition imprimée

Date de publication : 30 juin 2012

Pagination : 13-23

ISBN : 978-2-85831-200-9

ISSN : 0338-9316

\section{Référence électronique}

Rina Cohen Muller, «La presse hébraïque, un vecteur de l'entrée des Juifs dans la modernité », Yod [En ligne], 17 | 2012, mis en ligne le 22 octobre 2012, consulté le 08 juillet 2021. URL : http:// journals.openedition.org/yod/1575; DOI : https://doi.org/10.4000/yod.1575

Ce document a été généré automatiquement le 8 juillet 2021.

\section{(c) (1) (9)}

Yod est mis à disposition selon les termes de la Licence Creative Commons Attribution - Pas d'Utilisation Commerciale 4.0 International. 


\title{
La presse hébraïque, un vecteur de l'entrée des Juifs dans la modernité
}

The Press as a Vehicle in the Modernisation of the Jews

העיתונות כגורם מוביל בתהליך המודרניזציה של היהודים

\author{
Rina Cohen Muller
}

1 La presse hébraïque et son développement sont à la fois le produit et l'un des vecteurs de la Haskala. Les Lumières juives combinées avec une immigration importante ont radicalement modifié les modes de vie des Juifs européens et, par voie de conséquence, les pratiques sociales et culturelles des Juifs du monde entier.

De nombreuses études ont été consacrées à ce domaine dont les plus connues, les travaux de Kressel, Yardeni et Gilboa ${ }^{1}$, sont devenues une référence incontournable. Cependant, ces recherches se concentrent essentiellement sur les revues en tant que telles. Elles analysent, en dehors de tout rapport avec une conjoncture plus globale, les contenus et les thématiques des publications, les rapports entre les rédacteurs des différents titres. Ces travaux observent l'apport à la révolution de la langue et se satisfont de constater que les revues sont une source première pour la connaissance de l'histoire. En revanche, ce corpus n'a pas été exploité dans toute sa richesse en tant que formidable outil de communication et de source historique.

\section{Les débuts de la presse juive}

3 On situe l'origine de la presse juive au $\mathrm{XVII}^{\mathrm{e}}$ siècle à ce que l'on considère comme la première publication connue, la Gazeta de Amsterdam - en langue espagnole - parue aux Pays-Bas durant 27 ans à partir de 1674. Reprenant la formule de la Gazette d'Amsterdam (en français) qui diffuse des informations politiques et commerciales pour les négociants, elle est destinée aux commerçants juifs ayant fui l'Espagne et le Portugal ${ }^{2}$. Douze ans plus tard, d'août 1686 à décembre 1687, un éphémère journal en yiddish Dienstagishe un Fraytagishe Kuranten, avec un même objectif d'informations économiques, est diffusé dans la communauté ashkénaze de la métropole néerlandaise. 
Il s'agit là des premières publications qui diffusent des informations pour un public juif. On peut, par ailleurs, signaler que des commerçants juifs espagnols de Newport (Rhode Island) lisent la Gazeta. Il s'agit de journaux qui, par leur contenu principalement commercial et non spécifiquement communautaire, précèdent, par leur manière de voir le monde, de près d'un siècle les idées des Lumières ${ }^{3}$. Quelques autres revues, essentiellement consacrées à des responsa sur la loi religieuse et son application dans tous les domaines de la vie quotidienne sont créées à la même époque. Les plus connues paraissent aussi à Amsterdam à la fin du XVII ${ }^{e}$ et ensuite, à Venise au début du XVIII ${ }^{\mathrm{e}}$ siècle ${ }^{4}$. D'autres encore sont fondées au XIX ${ }^{\mathrm{e}}$ siècle. La première revue marquée par la Haskala en ses débuts est Qohelet musar (1750) que l'historien Heinrich Graetz attribue au fondateur des Lumières juives, Moses Mendelssohn. Il s'agit d'une sorte de guide philosophique avec un message humaniste, considéré comme une révolution dans la pensée juive - l'homme n'est pas uniquement sur terre pour vivre selon la loi divine, mais il existe aussi en soi, pour soi. En effet, le message fondateur de la Haskala préconise de rapprocher les Juifs des non-juifs et, au nom du rationalisme, l'acquisition du savoir afin de leur permettre de s'intégrer dans leur environnement. L'épanouissement de ce mouvement prend des formes multiples, dont notamment une approche du judaïsme comme objet d'étude, comme science raisonnée à part entière, ce qui au début du xix ${ }^{\mathrm{e}}$ siècle s'exprime par Hokhmat Israel ${ }^{5}$ avec sa composante, l'apprentissage de l'hébreu comme langue de culture.

4 C'est donc à côté d'ouvrages fondateurs de la Wissenschaft que se manifeste une nouvelle forme de diffusion de la culture juive et de communication, la presse avec près de 400 publications au cours de ce siècle. Elle participe de la mutation de l'attitude des Juifs envers le judaïsme. Progressivement, on ne le conçoit plus comme une pratique totalisante, mais comme une affaire relevant du domaine privé ou de la culture. Dans la Confédération germanique, en l'espace d'une génération, l'abandon des pratiques ancestrales du judaïsme modifie foncièrement le mode de vie des Juifs. Ils participent activement à la vie économique et à l'industrialisation naissante et, souvent, ils accèdent à la bourgeoisie allemande, avec tous ses attributs.

5 Au début du XIX ${ }^{e}$ siècle, les idées de modernisation du judaïsme atteignent la zone de résidence russe ${ }^{6}$ où les Juifs constituent $10 \%$ de l'ensemble de la population. Les maskilim russes tout en préconisant la modernisation, prennent leurs distances avec la Haskala allemande, y voyant ce qu'ils considèrent comme des risques d'abandon du judaïsme. Selon eux les problèmes matériels et moraux des Juifs sont la conséquence de la sclérose du judaïsme. Il s'agit donc, en premier lieu, de sortir les Juifs de la misère, tout particulièrement par l'éducation. Cette période donne naissance à l'épanouissement de la littérature hébraïque moderne au détriment du yiddish considéré à cette époque-là comme un " jargon ». Parallèlement, le tsar Alexandre II, dans le contexte de ses réformes tendant à l'émancipation de ses sujets, autorise certains Juifs ${ }^{7}$ à circuler librement et à s'installer en dehors de la zone de résidence, à avoir accès à l'éducation, à apprendre le russe, etc.

6 La naissance d'une presse spécifique participe largement à cette révolution culturelle qui transforme les réalités juives de fond en comble. Il s'agit, plus globalement, d'une nouvelle forme de communication, de culture, de diffusion des idées. Elle devient la plate-forme du débat public, se substituant à la place centrale qu'occupait la synagogue en tant qu'espace de rencontre et de culture. La circulation de l'information permet de tisser des liens entre les différentes communautés et les libère de l'isolement tout en les 
plaçant en contact direct avec la réalité sociétale environnante. Elle contribue également à préserver ces contacts alors que se manifestent une grande mobilité et parfois la dispersion de telle ou telle communauté. Dans ce même souffle, les revues et les journaux ont permis de rassembler des gens autour d'un message culturel et idéologique nouveau.

7 En même temps, des publications orthodoxes paraissent dès la deuxième moitié du XVII siècle notamment à Amsterdam et à Venise, puis en Allemagne, en Galicie et aux ÉtatsUnis. Ces revues et journaux se consacrent au combat contre les idées de la modernité.

Mais le plus grand nombre est celui des publications dites mixtes traitant à la fois de sujets juifs et de vulgarisation scientifique. La démarche pédagogique tend à faire comprendre que les Juifs font d'abord partie du pays dans lequel ils vivent, prioritairement à l'appartenance à une communauté religieuse. L'utilisation de l'hébreu en revanche exprime l'aspiration à l'appartenance à un groupe, compte tenu du fait que ces premières générations sorties de l'isolement du shtetl apprenaient la langue des pays dans lesquels elles vivaient. Les traductions en hébreu leur permettaient de faire la transition entre la culture juive et la culture générale.

\section{Les prémices d'une presse moderne}

9 L'année 1856 constitue un tournant dans le développement de la presse juive, avec la parution rapidement régulière des premiers hebdomadaires qui ouvrent la voie à la création de quotidiens. Le premier de ces hebdomadaires, Ha-Maggid (1856-1903), paraitt à Lyck (Prusse orientale) puis à Cracovie, suivent Ha-Melits (1860-1904, Odessa), HaCarmel (1860-1880, Vilno), Ha-Tsfira (1862-1931 Varsovie). On souligne souvent le nombre important de revues; environ 250 publications, uniquement en hébreu, peuvent être répertoriées jusqu'à la fin du xix ${ }^{e}$ siècle. Une soixantaine d'entre elles sont des livraisons uniques, 25 ne paraissent que deux fois. Leur véritable innovation se situe dans la place importante accordée aux points de vue, généralement sous forme d'articles éditoriaux, une pratique toute nouvelle. Une place importante est accordée à la littérature: romans traduits en hébreu, contes et nouvelles, critiques littéraires. Autre nouveauté, des informations ne concernant pas la sphère juive font leur apparition, essentiellement sous forme de nouvelles brèves souvent reprises et traduites de la presse généraliste locale. Cependant, l'essentiel de l'actualité demeure largement réservé aux nouvelles en provenance des communautés juives dans le monde. Souvent aussi, les différents articles sont repris de publications voisines, une pratique réciproque entre journaux juifs.

10 Dans ce siècle marqué par de vastes mouvements migratoires, par le début de la révolution industrielle et une mutation dans la vision du monde, cette presse permet aussi de rester en contact avec sa communauté d'origine tout en servant d'outil culturel pour ces Juifs qui accèdent à la bourgeoisie et qui n'ont pas ou plus les repères de la culture juive traditionnelle. En un premier temps lecture de loisir, la revue devient ainsi un nouveau pôle de référence pour ses lecteurs.

11 Ces journaux ont donc pour objet les transformations sociales illustrées, par exemple, par des productions littéraires. Mais l'enjeu fondamental porte sur les différentes conceptions de la Haskala, ses implications dans la transformation de la manière de vivre des Juifs et surtout, sur les changements dans la pratique religieuse et sur la place 
de cette dernière dans la vie quotidienne. Si Ha-Melits milite en faveur de réformes religieuses, Ha-Maggid, qui fut le premier à innover sa manière d'informer, se situe plus dans l'orthodoxie. Il s'oppose par exemple à l'émigration aux États-Unis considérée comme un péché, tandis que Ha-Melits et Ha-Tsfira ont une position plus modérée à l'égard de ce phénomène touchant l'ensemble des populations juives d'Europe de l'Est ${ }^{8}$. Mais à partir de la fin des années 1870 , à la veille de la première aliya, avec les premières acquisitions de terres en Palestine, la colonisation juive est considérée favorablement par les journaux juifs. Dernière nouveauté et non des moindres, les rédacteurs en chef de ces revues sont souvent eux-mêmes des figures de proue de la littérature hébraïque moderne.

\section{Une presse, une terre, une langue}

12 Les premiers journaux hébraïques de Jérusalem, Ha-Levanon et Ha-Havatselet, font leur apparition en 1863 dans un contexte géopolitique particulier. La Palestine devient l'un des principaux enjeux géostratégiques de la rivalité entre puissances européennes et russe, de la Question d'Orient et son corollaire, le projet de démantèlement de l'Empire ottoman. Dans le même temps, l'impact stabilisateur des Tanzimat ${ }^{9}$ et une croissance importante de la population juive en bouleversent les pratiques sociales. Essentiellement implantée Jérusalem, cette population passe de 2250 en 1800 (sur 9000 habitants) à 17000 en 1880 (sur 31 000). Du fait d'une forte immigration, les Juifs ashkénazes hassidiques - fuyant principalement la dégradation des conditions de vie en Europe mais aussi la Haskala dont, paradoxalement, des éléments importants prennent forme également dans la ville sainte - deviennent la majorité. Les Juifs de Jérusalem, à l'image des autres Hiérosolymites, sont divisés en communautés rivales souvent violemment hostiles entre elles. Ils ne ressentent pas le besoin de journaux, si ce n'est comme moyen de propagande vers la diaspora. Les communautés ashkénazes d'installation récente défendent âprement leur indépendance vis-à-vis de la population séfarade autochtone. La presse leur permet de faire connaître leur cause et d'exprimer leur opinion concernant les questions qui préoccupent les Juifs dans le reste du monde.

La naissance d'une presse hébraïque en Palestine est marquée par l'aspiration à préserver les caractéristiques traditionnelles de l'ancien yishuv dont l'activité essentielle consiste à étudier la Thora en comptant sur la charité (haluqa) pour vivre. Le débat ${ }^{10}$ au sein de la communauté ashkénaze, en passe de devenir majoritaire à Jérusalem, se situe non pas entre la Haskala et la Tradition mais entre les partisans de l'orthodoxie favorables à une certaine forme de mise à jour (par exemple le fait de travailler pour subvenir à ses besoins) - les Hassidim - et ceux qui affirment la nécessité du respect intégral de la manière de vivre de l'ancien yishuv, les Prushim ${ }^{11}$. C'est dans ce contexte que parait en février 1863 une première publication, celle des Prushim, Ha-Levanon ${ }^{12}$. Consacrée pour plus de la moitié aux questions religieuses relatives à la Terre d'Israël, cette publication se singularise par l'importance de sa rubrique éditoriale dont le contenu s'étale parfois sur plusieurs numéros. Le thème récurrent en est le devenir du yishuv fondé sur le devoir pour ses membres de se consacrer totalement à l'étude de la Loi. Dans ce sens, Ha-Levanon est le porte-parole d'un yishuv séculaire et immuable rejetant, de ce fait, l'immigration et la colonisation agricole. Cherchant à décourager définitivement la venue de Juifs européens et russes, Ha-Levanon décrit la Palestine comme un endroit sinistre où « des assassins et brigands 
rôdent toujours autour de nous » et où « l'Ismaélite abuse cruellement du Juif ». Par voie de conséquence, le journal s'oppose à toute initiative d'acquisition de terres qui appellerait les Juifs à se mettre au travail pour subvenir à leurs besoins ${ }^{13}$.

Cinq mois après Ha-Levanon, paraît Ha-Havatselet édité par des Hassidim. Ces derniers (Israel Beck, puis Dov Frumkin) soutiennent l'idée que les Juifs du yishuv doivent s'engager dans l'activité productive. Leur journal présente une image bien plus pacifique de la Palestine. Il diffuse des informations relatives à la Palestine, à l'Empire ottoman et aux investissements locaux de Moses Montefiore, ainsi qu'à toute nouveauté ou progrès technique et scientifique.

Moins d'un an après leur parution, les deux journaux sont interdits par les autorités ottomanes. N'ayant pas l'autorisation officielle de paraître, ils s'étaient réciproquement dénoncés. Ha-Levanon émigre à Paris puis à Mayence, tandis que Ha-Havatselet dont le rédacteur en chef connaît mieux les rouages de l'administration ottomane, obtient un firman et reparaît à Jérusalem un an plus tard avec, pour un temps, des éditions en judéo-espagnol et en yiddish.

Dès l'installation à partir de 1882 de colonies juives, $\mathrm{Ha}$-Havatselet soutient le processus avec ses implications socio-économiques. La critique du fonctionnement du yishuv en général est certes présente, mais modérée. Le journal condamne les dissensions entre communautés orthodoxes. Il dénonce l'inscription par des Juifs de leurs enfants dans les écoles des missions chrétiennes. Il condamne aussi l'abandon croissant de la religion dans les colonies juives et l'utilisation de l'hébreu comme langue parlée. Dans le même temps, Ha-Havatselet s'engage en faveur de la modernisation de l'ancien yishuv tout en restant vigilant. Ce journal demeure l'une des principales sources d'information sur la Palestine, notamment sur sa population juive.

\section{L'apport fondateur de Ben Yehuda}

Alors qu'il assume la fonction de rédacteur en chef de Ha-Havatselet au cours de l'année 1882, Eliezer Ben Yehuda, généralement considéré comme à l'origine de l'hébreu moderne, signe un article demandant l'aide de l'Alliance israélite universelle. Il regrette que cette dernière, après avoir dans un premier temps favorisé la venue de Juifs en Palestine, préfère encourager l'émigration en Amérique. Tout au long de son intérim, Ben Yehuda informe ses lecteurs de l'évolution de l'immigration juive. Il les alerte sur la pénurie croissante de logements ainsi que sur la famine qui fait des nouveaux arrivants une proie pour les missions chrétiennes qui leur proposent de la nourriture en échange souvent d'une conversion.

Malgré sa relative ouverture d'esprit, Frumkin désapprouve la démarche de Ben Yehuda qui lui paraît trop osée. Non seulement ce dernier essaie d'imposer les pratiques journalistiques qu'il a connues à Paris mais ses écrits semblent privilégier l'idée nationale, tandis que Frumkin donne la priorité au combat contre l'obscurantisme.

La fondation en 1885 de Ha-Tsvi par Ben Yehuda est l'occasion pour celui-ci de mettre en œuvre ses conceptions novatrices. Sa publication marque un tournant par la clarté de ses articles écrits en langage journalistique direct en rupture avec le style ampoulé et pompeux des journaux juifs. Il s'agit avant tout d'un journal d'information destiné à la population juive locale et où l'actualité non juive prend sa place. Rédigés en hébreu, 
les textes sont le support de mots nouveaux, souvent expliqués en note avec parfois leurs équivalents en français ou en allemand. Ces notes forment l'embryon de son futur dictionnaire. Dès le premier numéro, le journal met en cause les comportements et les habitudes des Juifs de Jérusalem et leur dépendance envers la haluqa. En réclamant son abolition, il provoque l'hostilité des rabbins qui vont jusqu'à le frapper d'anathème. Il dénonce la saleté des quartiers juifs, l'ignorance et les superstitions de leurs habitants. Il dénonce également la tutelle gestionnaire du baron de Rothschild sur les colonies, les privant de toute initiative. Comme dans le reste de la presse hébraïque, un espace est réservé aux romans-feuilletons de la littérature mondiale.

\section{La presse hébraïque devient nationale}

20 L'histoire de la presse hébraïque en Palestine est enchevêtrée dans l'histoire du sionisme et de la formation de la société civile juive moderne. Cette presse possède la double particularité d'avoir été au service exclusif de la population juive de la province ottomane et d'être, aujourd'hui encore, essentiellement un média d'opinion. Jusqu'aux années 1930, la presse du yishuv ne fait que peu de cas de la population autochtone non juive. Elle n'en donne qu'une vision partielle et exotique à l'instar de celle des grandes puissances.

21 En 1907, la presse hébraïque palestinienne se stratifie avec la parution de la première publication politique, l'hebdomadaire de Ha-po'el ha-tsa'ir - sioniste socialiste - qui porte le même nom. En 1910 est fondé Ahdut, l'organe de Po'ale Tsion-socialiste sioniste. Ces deux organisations qui sont à l'origine du mouvement ouvrier fusionnent pour constituer ultérieurement le mouvement travailliste. Ces deux journaux s'opposent à celui de Ben Yehuda et plus tard à la publication de son fils, Doar ha-yom, qu'ils qualifient de journal à scandale. À la même époque parait une première revue séfarade Ha-Herut (1909-1917). Avec Moria, les orthodoxes cherchent à retrouver un lectorat. Enfin on observe l'apparition de presses enfantine, humoristique et littéraire ${ }^{14}$. Les principaux journaux, et notamment les organes de partis, contribuent à la politisation de la population du nouveau yishuv. Dans cet ensemble, une exception remarquable, tant par sa conception que par sa pérennité, est la parution à partir de juin 1919 du quotidien Hadshot Haaretz qui devient six mois plus tard Haaretz. Ce journal est en quelque sorte le symbole - qui jusqu'à aujourd'hui demeure unique en Israël d'une publication non affiliée ou liée à une organisation politique.

On peut, en guise de conclusion, affirmer qu'avec sa presse, la culture hébraïque de la Palestine prend le dessus sur les différents centres hébraïques de la diaspora (Berlin, Odessa, Varsovie, etc.). À partir des années 1920, c'est principalement à Tel-Aviv que s'élabore cette vision nouvelle d'une culture hébraïque autochtone avec ses graines nationales, tandis que dans la diaspora fleurit une culture juive multiple dans la langue des pays de résidence ainsi que dans les langues juives ${ }^{15}$. 


\section{BIBLIOGRAPHIE}

Early Hebrew Newpapers: http://www.jnul.huji.ac.il/dl/newspapers/index1024.html

Historical Jewish Press: http://www.jpress.org.il/view-hebrew.asp

Qohelet Musar: http://hebrewbooks.org/42578

GILBOA, Menucha (1992), לקסיקון העיתונות העברית במאות השמונה-עשרה והתשע-עשרה Lexique de la presse hébraïque au XVIII et XIX ${ }^{e}$ siècles, Jérusalem, Mosad Bialik.

KOUTS, Gideon (1993), La Naissance de la presse hébraïque moderne : Les grands périodiques en Europe, 1856-1886, Paris, Maisonneuve \& Larose.

KRESSEL, Gezel (1964), תולדות העיתונות העברית בא" Histoire de la presse hébraïque en Eretz-Israel, Jérusalem, Hasifriya hatsionit.

PATAI, Raphael (1977), The Jewish Mind, Michigan, Wayne University Press.

SHAVIT, Zohar (1999), « Le développement des périodiques et de la presse » in Moshe Lissak (ed.), תולדות היישוב היהודי בא"י מאז העלייה הראשונה - בנייתה של תרבות עברית Histoire du yishuv en Eretz-Israel - La construction d'une culture hébraïque, Jérusalem, Mossad Bialik, vol. I.

WILKE, Carsten (2007), Histoire des Juifs portugais, Paris, Chadeigne, Librairie portugaise.

YARDENI, Galia (1969),1904-1863 העיתונות העברית בארץ-ישראל בשנים La Presse hébraïque en EretzIsrael (1863-1904), Tel-Aviv, Hakibbutz Hameuchad.

\section{NOTES}

1. Kressel Gezel (1964); Yardeni Galia (1969) ; Gilboa Menucha (1992).

2. Wilke Carsten (2007), p. 175 ; Patai Raphael (1977), p. 230-231.

3. Selon C. Wilke, il s'agissait également d'une mesure de précaution pour ne pas mettre en danger les lecteurs vivant dans le Sud-Ouest français.

4. Pri 'ets ha-hayyim (1691-1807), 953 responsa en 13 volumes, édité par la communauté séfarade d'Amsterdam ; Bikkure qatsir (1715), responsa des élèves et maîtres du Talmud Thora de Ferrare.

5. Wissenschaft des Judentums devenant plus tard la « science du judaïsme».

6. Couvrant une superficie d'environ un million de $\mathrm{km}^{2}$.

7. Ceux qui exercent un métier, par exemple.

8. Rappelons qu'à la fin du XIX $x^{\mathrm{e}}$ siècle, la population juive en Russie est estimée à cinq millions de personnes. Au cours de la période 1870-1914, deux millions de Juifs s'installent aux États-Unis.

9. Les grandes réformes qui ont pour but la modernisation de l'État ottoman (1839-1876).

10. G. Kressel, op. cit., p. 30.

11. Nom donné aux disciples du Gaon de Vilna, et à leurs descendants, qui se sont installés en Palestine au début du XIX ${ }^{\mathrm{e}}$ siècle.

12. L'éditeur, Yehiel Bril, originaire de Tolchin (Podolie - Ukraine) rejoint la communauté des Prushim à Jérusalem.

13. S'opposant ainsi aux premières acquisitions de terres par Ha-hevra le-yishuv ha-arets (1860-1865) fondée par le rabbin Alkalay, considéré comme l'un des présionistes traditionnels.

14. Zohar Shavit, (1999), p. 123-198.

15. Ibid. 


\section{RÉSUMÉS}

La presse hébraïque et son développement sont à la fois le produit et l'un des vecteurs de la Haskala. Les Lumières juives marquent l'entrée des Juifs dans la modernité. Ces journaux émergent alors que le monde juif est en ébullition. Jusqu'à la fin du XIX ${ }^{\mathrm{e}}$ siècle, on compte environ 250 titres. La presse devient rapidement un mode d'expression majeur qui s'étend à l'ensemble du judaïsme européen, essentiellement en Europe de l'Est. Dans le même temps, des journaux apparaissent en Palestine, une presse qui se place également dans la mouvance des Lumières, avec cependant une différence : elle prend la voie d'une presse nationale eretz-israélienne.

The Hebrew press and its development are the product and, at the same time, one of the vectors of the Haskala. The Jewish Enlightenment marks the entry of the Jews into modernity. These newspapers appear in a period when the Jewish world is in effervescence. We count approximately 250 titles until the end of the 19th century. The press quickly becomes a major mode of expression which extends to the whole European Judaism, essentially in Eastern Europe. At the same time, newspapers appear in Palestine, forming a press, which evolves also within the orbit of the Enlightenment, however with one important distinctive feature: it becomes an eretzIsraeli national press.

INDEX

\section{מילות מפתח}

הלבנון, החבצלת, השכלה, עיתונות עברית, עיתונות יהודית:

Keywords : Ha-Levanon, Ha-Havatselet, haskala, Hebrew press, Jewish press, literature

Mots-clés : Ha-Levanon, Ha-Ḥavatselet, haskala, presse hébraïque, presse juive

Thèmes : littérature 